\title{
Article \\ Fourier Transform for Locally Integrable Functions with Rotational and Dilation Symmetry
}

\author{
Jerzy A. Przeszowski *(D), Elżbieta Dzimida-Chmielewska (i) and Jan L. Cieśliński (1) \\ Faculty of Physics, University of Bialystok, ul. Ciołkowskiego 1L, 15-245 Bialystok, Poland; \\ edzimida@uwb.edu.pl (E.D.-C.); j.cieslinski@uwb.edu.pl (J.L.C.) \\ * Correspondence: j.przeszowski@uwb.edu.pl
}

check for updates

Citation: Przeszowski, J.A.; Dzimida-Chmielewska, E.; Cieśliński, J.L. Fourier Transform for Locally Integrable Functions with Rotational and Dilation Symmetry. Symmetry 2022, 14, 241. https://doi.org/ $10.3390 /$ sym 14020241

Academic Editor: Vladimir Al. Osipov

Received: 20 December 2021 Accepted: 22 January 2022 Published: 26 January 2022

Publisher's Note: MDPI stays neutral with regard to jurisdictional claims in published maps and institutional affiliations.

Copyright: (C) 2022 by the authors Licensee MDPI, Basel, Switzerland. This article is an open access article distributed under the terms and conditions of the Creative Commons Attribution (CC BY) license (https:// creativecommons.org/licenses/by/ $4.0 /)$.

\begin{abstract}
The Fourier transform for slowly increasing functions is defined by the Parseval equation for tempered distributions. This definition was supplemented by a novel method of performing practical calculations by computing the Fourier transform for a suitably tempered function and then by integration by parts. The application of this method is illustrated both for the toy case, in which the function is integrable, so its Fourier transform can also be computed using the standard formula, and for the case of Coulomb-like potentials, which are only locally integrable functions. All of them have spherical symmetry, and two of them additionally have dilation symmetry. The proposed novel method does not violate these symmetries at any stage of the calculation.
\end{abstract}

Keywords: Fourier transform; tempered distribution; Parseval equation; rotational symmetry; dilation symmetry; modified Bessel function; exponential integral function

\section{Introduction}

The Fourier transform (FT), defined for an integrable function, i.e., $\psi \in L^{1}\left(\mathbb{R}^{3}\right)$ as [1]

$$
\mathscr{F}\{\psi\}(\boldsymbol{k})=\int_{\mathbb{R}^{3}} e^{-i \boldsymbol{k} \cdot \boldsymbol{x}} \psi(\boldsymbol{x}) d^{3} \boldsymbol{x}, \quad \mathscr{F}^{-1}\{\psi\}(\boldsymbol{x})=\int_{\mathbb{R}^{3}} e^{i \boldsymbol{k} \cdot \boldsymbol{x}} \psi(\boldsymbol{k}) \frac{d^{3} \boldsymbol{k}}{(2 \pi)^{3}}
$$

is a very useful concept that simplifies solutions of many problems in physical sciences, engineering, etc. Unfortunately, for several important physical quantities, the above definition cannot be applied, e.g., for the Coulomb potential $V_{\mathcal{c}}(\boldsymbol{x})=\frac{1}{r}$, with $r=|\boldsymbol{x}|=\sqrt{\boldsymbol{x} \cdot \boldsymbol{x}}$, which plays an important role in the description of electrostatic [2] and gravitational interactions, but evidently $V_{c} \notin L^{1}\left(\mathbb{R}^{3}\right)$. Not surprisingly, the standard definition of FT, when integrated over spherical angles in the position space $\mathbb{R}^{3}$, leads to an ill-defined integral

$$
\mathscr{F}\left\{V_{c}\right\}(\boldsymbol{k})=\int_{\mathbb{R}^{3}} e^{-i \boldsymbol{k} \cdot \boldsymbol{x}} V_{c}(\boldsymbol{x}) d^{3} \boldsymbol{x}=\frac{4 \pi}{k} \int_{\mathbb{R}_{+}} \sin (k r) d r,
$$

where $\mathbb{R}_{+}=\{x \in \mathbb{R}: x \geq 0\}$ and $k=|k|$. To eliminate this deficiency, a damping factor $e^{-\alpha r}$, with $\Re \alpha>0$, is commonly added; for example, see [3-5], which converts the long-range Coulomb potential into the short-range Yukawa potential $V_{\alpha}(x)$

$$
V_{c}(\boldsymbol{x})=\frac{1}{r} \rightarrow V_{\alpha}(\boldsymbol{x})=\frac{e^{-\alpha r}}{r} \Longrightarrow \mathscr{F}\left\{V_{\alpha}\right\}(\boldsymbol{k})=\frac{4 \pi}{k} \int_{\mathbb{R}_{+}} d r \sin (k r) e^{-\alpha r}=\frac{4 \pi}{k^{2}+\alpha^{2}} .
$$

Then, it is argued that the FT of the Coulomb potential $V_{c}$ can be defined by the limit $\alpha \searrow 0$ of $\mathscr{F}\left\{V_{\alpha}\right\}$, which is based on the analogy

$$
V_{c}(\boldsymbol{x})=\lim _{\alpha \searrow 0} V_{\alpha}(\boldsymbol{x}) \Longrightarrow \mathscr{F}\left\{V_{c}\right\}(\boldsymbol{k}):=\lim _{\alpha \searrow 0} \mathscr{F}\left\{V_{\alpha}\right\}=\frac{4 \pi}{k^{2}} .
$$

The advantage of such a procedure is that the spherically symmetric Coulomb potential is replaced by a spherically symmetric Yukawa potential, which means that rotational sym- 
metry remains unbroken. However, this does not apply to the dilation symmetry associated with the transformation $x \rightarrow x^{\prime}=\lambda x$, where the Coulomb potential is dilational covariant

$$
x \rightarrow x^{\prime}=\lambda x \Longrightarrow V_{c}(x) \rightarrow V_{c}^{\lambda}(x)=V_{c}(\lambda x)=\frac{1}{\lambda} V_{c}(x),
$$

whereas the Yukawa potential explicitly breaks this symmetry. It seems obvious that any damping factor will break the dilation symmetry, so you have to invent a completely different computational procedure to preserve the dilation symmetry. This trouble of calculating the FT for the Coulomb potential can be avoided by saying that this FT is determined uniquely by the Poisson equation [6], but we did not pursue such reasoning further.

In this work, we present a novel method for calculating FT, which, when applied directly to the Coulomb potential, will reproduce the above result but without recourse to the Yukawa potential, so that both rotational and dilational symmetries remain intact at each stage of the procedure. Here, the dilational symmetry represents a physical symmetry that should be easily controlled at each stage of the computation. For more complicated cases, recovering such symmetry in the final result can lead to quite serious problems such as noncommutativity of limits, etc.). It will use the distributions with the test functions of the Schwartz class $\mathscr{S}^{\prime}\left(\mathbb{R}^{3}\right)$, which due to the Parseval equation for the tempered distributions will lead to FT in the sense of distributions. For checking the consistency of this method, one may take a toy case $W(x)=\frac{1}{x^{2}} \in L^{1}\left(\mathbb{R}^{3}\right)$, which gives the same FT, as the standard formula (1). Next, we consider the Coulomb-like potentials, which are slow-growing functions and thus have no standard FT, according to (2). First, we considered the softened Coulomb potential with a decaying exponential term $V_{p}(x)=\frac{1}{r} e^{-c / r}$, with $c>0$, [7], which vanishes for $r \rightarrow 0$. Then, we studied the soft-core Coulomb potential $U_{a}(x)=\frac{1}{\sqrt{r^{2}+a^{2}}}$, with an arbitrary parameter $a \in \mathbb{R}_{+}$[8], which is a special case of a generic soft-core Coulomb potential $U_{a, q}(x)=\frac{1}{\left(r^{q}+a^{q}\right)^{1 / q}}$, for $q \geq 1$ [9-11], which is used for modeling potentials in atomic and molecular physics. At last, we considered the pure Coulomb potential $V_{c}$, which has both rotational and dilation symmetry, unlike the previous two cases, which have only rotational symmetry. Our novel method is shown to be equivalent to the standard one, with the exponential damping factor, when the distributional derivatives are the standard derivatives-this is presented in the Appendix A. Finally, we mention how our method can be compared to the one used in [12], where dilation symmetry is used as a key step in the computation.

\section{Calculation of Distributional FT-Methods}

We begin by recalling some important properties of the Fourier transform and tempered decompositions that can be found in the vast literature, where the fundamental monograph by Gelfand and Shilov (see [12] pp. 71-74 and pp. 190-200) should be mentioned, but we can also select some items relevant to physics: [13-15]. The standard Fourier transform defined by (1) for integrable functions in $L^{1}\left(\mathbb{R}^{3}\right)$ is a continuous and bounded function on $\mathbb{R}^{3}$, and it vanishes at infinity. For $\phi, \psi \in L^{1}\left(\mathbb{R}^{3}\right)$ one finds $\psi \mathscr{F}\{\phi\} \in L^{1}\left(\mathbb{R}^{3}\right)$ and $\phi \mathscr{F}\{\psi\} \in L^{1}\left(\mathbb{R}^{3}\right)$, so the Parseval theorem

$$
\int_{\mathbb{R}^{3}} \phi(x) \mathscr{F}\{\psi\}(x) d x=\int_{\mathbb{R}^{3}} \psi(y) \mathscr{F}\{\phi\}(y) d y,
$$

follows from Fubbini's theorem, where we used a compact notation for $x, y \in \mathbb{R}^{3}$. Evidently, this Parseval theorem is valid also for the Schwartz test function $\phi \in \mathscr{S}\left(\mathbb{R}^{3}\right)$, because $\mathscr{S}$ is a dense subspace of $L^{p}$ with $1 \leq p<\infty$. Moreover in this case both sides of (6) are the regular distributions $\mathscr{S}^{\prime}\left(\mathbb{R}^{3}\right)$. This is a consequence of the fact that the Fourier transform is a bijection map $\mathscr{F}: \mathscr{S}\left(\mathbb{R}^{3}\right) \rightarrow \mathscr{S}\left(\mathbb{R}^{3}\right)$ from the space $\mathscr{S}\left(\mathbb{R}^{3}\right)$ unto itself, and hence $\mathscr{F}\{\phi\} \in \mathscr{S}\left(\mathbb{R}^{3}\right)$. The situation changes for $\phi \in \mathscr{S}\left(\mathbb{R}^{3}\right)$ and $L_{\text {loc }}^{1}\left(\mathbb{R}^{3}\right) \ni \psi \notin L^{1}\left(\mathbb{R}^{3}\right)$, where 
$\mathscr{F}\{\psi\}$ cannot be given by the standard definition (1), so we cannot calculate the left-hand side in (6). In contrast, the right-hand side in (6) is a tempered distribution $\mathscr{S}^{\prime}\left(\mathbb{R}^{3}\right)$

$$
\psi[\mathscr{F}\{\phi\}]=\int_{\mathbb{R}^{3}} \psi(y) \mathscr{F}\{\phi\}(y) d y,
$$

provided $\psi$ is a slow-growing function, i.e., it has polynomial growth as $|x| \rightarrow \infty$, which gives $\psi \mathscr{F}\{\phi\} \in \mathscr{S}\left(\mathbb{R}^{3}\right)$. This allows us to reinterpret (6) as a definition of distributional FT in the sense of distributions $\mathscr{S}^{\prime}\left(\mathbb{R}^{3}\right)$.

Definition 1. For a slow-growing locally integrable function $\psi$ its distributional Fourier transform $\mathscr{F}_{D}\{\psi\}$ is given by the relation for tempered distributions $\mathscr{S}^{\prime}\left(\mathbb{R}^{3}\right)$

$$
\int_{\mathbb{R}^{3}} \phi(x) \mathscr{F}_{D}\{\psi\}(x) d x=\int_{\mathbb{R}^{3}} \psi(y) \mathscr{F}\{\phi\}(y) d y, \quad \forall \phi \in \mathscr{S}\left(\mathbb{R}^{3}\right) .
$$

This equation will be called the Parseval equation for the distributional FT or the Parseval equation for short. Evidently if $\psi \in L^{1}\left(\mathbb{R}^{3}\right)$, then its distributional Fourier transform $\mathscr{F}_{D}\{\psi\}$ coincides with the standard one $\mathscr{F}\{\psi\}$ given by (1).

Before we move on to practical computations with the help of (8), we can slightly change the notation for the Schwartz test function by introducing $\mathscr{S}\left(\mathbb{R}^{3}\right) \ni \bar{\phi}_{\mathscr{F}}:=\mathscr{F}\{\phi\}$, which implies that $\phi=\mathscr{F}^{-1}\left\{\bar{\phi}_{\mathscr{F}}\right\}=(2 \pi)^{-3} \overline{\mathscr{F}\left\{\phi_{\mathscr{F}}\right\}}$, where the bar denotes the complex conjugate. Thus, we may re-express (7) and (8) as

$$
\begin{aligned}
& \psi\left[\bar{\phi}_{\mathscr{F}}\right]=\int_{\mathbb{R}^{3}} \psi(\boldsymbol{x}) \bar{\phi}_{\mathscr{F}}(\boldsymbol{x}) d^{3} \boldsymbol{x}, \\
& \left.\int_{\mathbb{R}^{3}} \overline{\mathscr{F}\{\phi \mathscr{F}}\right\}(\boldsymbol{k}) \mathscr{F}_{D}\{\psi\}(\boldsymbol{k}) \frac{d^{3} \boldsymbol{k}}{(2 \pi)^{3}}=\int_{\mathbb{R}^{3}} \psi(\boldsymbol{x}) \bar{\phi}_{\mathscr{F}}(\boldsymbol{x}) d^{3} \boldsymbol{x}, \quad \forall \bar{\phi}_{\mathscr{F}} \in \mathscr{S}\left(\mathbb{R}^{3}\right),
\end{aligned}
$$

with explicit notation for vectors $x, k \in \mathbb{R}^{3}$. Furthermore, in the following discussion, without loss of generality, we can drop the subscript in $\phi_{\mathscr{F}}=\phi$. The calculation of $\mathscr{F}_{D}\{\psi\}$ is based on the analysis of the tempered distribution $\psi[\bar{\phi}]$, and the standard procedure starts with a suitable modification of $\psi[\bar{\phi}]$, where a damping factor is inserted under the integral sign. Such a calculation can be illustrated using the Coulomb potential $\psi=V_{c} \notin L^{1}\left(\mathbb{R}^{3}\right)$ as an example, where relations (9) and (10) look as

$$
V_{c}[\bar{\phi}]=\int_{\mathbb{R}^{3}} V_{c}(\boldsymbol{x}) \bar{\phi}(\boldsymbol{x}) d^{3} \boldsymbol{x}=\int_{\mathbb{R}^{3}} \overline{\mathscr{F}\{\phi\}}(\boldsymbol{k}) \mathscr{F}_{D}\left\{V_{c}\right\}(\boldsymbol{k}) \frac{d^{3} \boldsymbol{k}}{(2 \pi)^{3}}
$$

However, for the tempered distribution $\psi[\bar{\phi}]=V_{c}[\bar{\phi}]$ we can introduce a damping factor $e^{-\alpha r}, \alpha>0$ and calculate using the complex conjugation of (1) for $\bar{\phi}(\boldsymbol{x})$,

$$
\begin{aligned}
V_{c}[\bar{\phi}] & =\lim _{\alpha \searrow 0} \int_{\mathbb{R}^{3}} V_{c}(\boldsymbol{x}) e^{-\alpha r} \bar{\phi}(\boldsymbol{x}) d^{3} \boldsymbol{x} \\
& =\lim _{\alpha \searrow 0} \int_{\mathbb{R}^{3}} V_{c}(\boldsymbol{x}) e^{-\alpha r}\left[\int_{\mathbb{R}^{3}} e^{-i \boldsymbol{k} \cdot \boldsymbol{x} \overline{\mathscr{F}}\{\phi\}}(\boldsymbol{k}) \frac{d^{3} \boldsymbol{k}}{(2 \pi)^{3}}\right] d^{3} \boldsymbol{x} \\
& =\lim _{\alpha \searrow 0} \int_{\mathbb{R}^{3}} \overline{\mathscr{F}\{\phi\}}(\boldsymbol{k})\left[\int_{\mathbb{R}^{3}} V_{c}(\boldsymbol{x}) e^{-\alpha r} e^{-i \boldsymbol{k} \cdot \boldsymbol{x}} d^{3} \boldsymbol{x}\right] \frac{d^{3} \boldsymbol{k}}{(2 \pi)^{3}} \\
& =\lim _{\alpha \searrow 0} \int_{\mathbb{R}^{3}} \overline{\mathscr{F}\{\phi\}}(\boldsymbol{k}) \mathscr{F}\left\{V_{\alpha}\right\}(\boldsymbol{k}) \frac{d^{3} \boldsymbol{k}}{(2 \pi)^{3}}=\lim _{\alpha \searrow 0} \int_{\mathbb{R}^{3}} \overline{\mathscr{F}\{\phi\}}(\boldsymbol{k}) \frac{4 \pi}{k^{2}+\alpha^{2}} \frac{d^{3} \boldsymbol{k}}{(2 \pi)^{3}},
\end{aligned}
$$

where we can switch the order of integrations by Fubini's theorem, because $V_{\alpha}(\boldsymbol{x})=V_{c}(\boldsymbol{x}) e^{-\alpha r} \in L^{1}\left(\mathbb{R}^{3}\right)$ and take $\mathscr{F}\left\{V_{\alpha}\right\}$ from (3). Then, for the last convergent integral, we can insert the limit $\alpha \searrow 0$ under the sign of the integral, thus reaching the 
left-hand side of the Parseval equation in the form (10) and yielding the distributional FT for the Coulomb potential $\mathscr{F}_{D}\left\{V_{c}\right\}$.

$$
V_{c}[\mathscr{F}\{\phi\}]=\int_{\mathbb{R}^{3}} \overline{\mathscr{F}\{\phi\}}(\boldsymbol{k}) \frac{4 \pi}{k^{2}+\alpha^{2}} \frac{d^{3} \boldsymbol{k}}{(2 \pi)^{3}} \Longrightarrow \mathscr{F}_{D}\left\{V_{c}\right\}=\frac{4 \pi}{k^{2}} .
$$

Accordingly, the ad hoc calculations presented in the introduction are closely related to the standard calculation of the distributional FT. Therefore, the previous drawbacks mentioned in the introduction also apply to this method.

We propose a different computational procedure, (a comparison of these two computational methods is presented in Appendix $\mathrm{A}$ ), that relies on specific properties of Schwartz test functions $\phi(x) \in \mathscr{S}\left(\mathbb{R}^{3}\right)$, namely, $\left(r^{2}+M^{2}\right)^{n} \phi(x) \in \mathscr{S}\left(\mathbb{R}^{3}\right)$, where $n \in \mathbb{N}$ and $M \in \mathbb{R}_{+}$ were chosen arbitrarily [14] (also see pp. 16-17 in [12]). From the definition (1), one checks the formula for FT: $\mathscr{F}\left\{\left(r^{2}+M^{2}\right)^{n} \phi\right\}(\boldsymbol{k})=\left(M^{2}-\nabla_{\boldsymbol{k}}^{2}\right)^{n} \mathscr{F}\{\phi\}(\boldsymbol{k}) \in \mathscr{S}\left(\mathbb{R}^{3}\right)$, where $\nabla_{\boldsymbol{k}}^{2}$ is the Laplace operator in the space of $k \in \mathbb{R}^{3}$. All this leads to the tempered distribution

$$
\begin{aligned}
& \psi[\bar{\phi}]=\int_{\mathbb{R}^{3}} \psi(\boldsymbol{x}) \bar{\phi}(\boldsymbol{x}) d^{3} \boldsymbol{x}=\int_{\mathbb{R}^{3}} \frac{\psi(\boldsymbol{x})}{\left(r^{2}+M^{2}\right)^{n}}\left(r^{2}+M^{2}\right)^{n} \bar{\phi}(\boldsymbol{x}) d^{3} \boldsymbol{x}, \\
& =\int_{\mathbb{R}^{3}}\left(M^{2}-\nabla_{k}^{2}\right)^{n} \overline{\mathscr{F}\{\phi\}}(\boldsymbol{k}) \mathscr{F}\left\{\frac{\psi}{\left(r^{2}+M^{2}\right)^{n}}\right\}(\boldsymbol{k}) \frac{d^{3} \boldsymbol{k}}{(2 \pi)^{3}},
\end{aligned}
$$

where we can take $n \in \mathbb{N}_{0}$ and $M \in \mathbb{R}_{+}$arbitrarily, provided that $\psi(\boldsymbol{x}) /\left(r^{2}+M^{2}\right)^{n} \in L^{1}\left(\mathbb{R}^{3}\right)$. Then, one can integrate by parts with respect to $k$, which gives the final equivalent form, which can be compared with the Parseval equation for the distributional FT (10)

$$
\psi[\phi]=\int_{\mathbb{R}^{3}} \overline{\mathscr{F}\{\phi\}}(\boldsymbol{k})\left(M^{2}-\bar{\nabla}_{\boldsymbol{k}}^{2}\right)^{n} \mathscr{F}\left\{\frac{\psi}{\left(r^{2}+M^{2}\right)^{n}}\right\}(\boldsymbol{k}) \frac{d^{3} \boldsymbol{k}}{(2 \pi)^{3}},
$$

where $\bar{\nabla}_{k}^{2}=\sum_{i=1}^{3} D_{i} D_{i}$ with the distributional partial derivatives $D_{i}$ in $\mathbb{R}^{3}$.

Lemma 2. If $L_{\text {loc }}^{1}\left(\mathbb{R}^{3}\right) \ni \psi \notin L^{1}\left(\mathbb{R}^{3}\right)$ and it has a polynomial growth for $|x| \rightarrow \infty$ and $\psi /$ $\left(r^{2}+M^{2}\right)^{n} \in L^{1}\left(\mathbb{R}^{3}\right)$ with appropriate choice of $n$ and $M$, then its distributional FT is given in the sense of distributions $\mathscr{S}^{\prime}\left(\mathbb{R}^{3}\right)$ by

$$
\mathscr{F}_{D}\{\psi\}(\boldsymbol{k})=\left(M^{2}-\bar{\nabla}^{2}\right)^{n} \mathscr{F}\left\{\psi /\left(r^{2}+M^{2}\right)^{n}\right\}(\boldsymbol{k}) .
$$

In case of rotational symmetry one has $\psi(x)=\psi(r)$, so the integral over the unit sphere embedded in $\mathbb{R}^{3}$ can be easily performed done leading to the result, which depends only on the radial variable $k=|k|$

$$
\mathscr{F}\left\{\frac{\psi}{\left(r^{2}+M^{2}\right)^{n}}\right\}(\boldsymbol{k})=\int_{\mathbb{R}^{3}} \frac{e^{-i \boldsymbol{k} \cdot \boldsymbol{x}} \psi(r)}{\left(r^{2}+M^{2}\right)^{n}} d^{3} x=\frac{4 \pi}{k} \int_{\mathbb{R}_{+}} \frac{r \sin (k r) \psi(r)}{\left(r^{2}+M^{2}\right)^{n}} d r .
$$

This in turn allows us to move on to integrals with respect to $k \in \mathbb{R}^{3}$, which can be expressed as the radial integral

$$
\psi[\bar{\phi}]=\int_{\mathbb{R}_{+}}\left(M^{2}-\partial_{k}^{2}\right)^{n}\left[k \overline{\mathscr{F}_{\text {rad }}\{\phi\}}(k)\right]\left[\int_{\mathbb{R}_{+}} \frac{r \sin (k r) \psi(r)}{\left(r^{2}+M^{2}\right)^{n}} d r\right] \frac{d k}{2 \pi^{2}},
$$

where the radial FT $\overline{\mathscr{F}_{\text {rad }}\{\phi\}}$ is defined as the integral over the unit sphere embedded in $\mathbb{R}^{3}$

$$
\overline{\mathscr{F}_{\text {rad }}\{\phi\}}(k):=\int_{\Omega_{3}} \overline{\mathscr{F}\{\phi\}}(\boldsymbol{k}) d \omega_{\boldsymbol{k}}=\frac{4 \pi}{k} \int_{\mathbb{R}^{3}} \sin (k r) \bar{\phi}(\boldsymbol{x}) d^{3} \boldsymbol{x} .
$$


where $d \omega_{k}$ is the hypersurface element on the unit sphere, and $\Omega_{3}=4 \pi$ is the hypersurface area of the unit sphere in $\mathbb{R}^{3}$ (see [12] p. 71). (Evidently $\overline{\mathscr{F}_{\text {rad }}\{\phi\}}(k)$ is the mean value of $\overline{\mathscr{F}_{\text {rad }}\{\phi\}}(\boldsymbol{k})$ on the sphere of radius $k$ (see [12] p. 71), and one may express (18) by means of the mean value of $\bar{\phi}(x)$ on the sphere of radius $k$

$$
\overline{\phi_{\text {rad }}}(r):=\int_{\Omega_{3}} \bar{\phi}(x) d \omega \Longrightarrow k \overline{\mathscr{F}_{\text {rad }}\{\phi\}}(k)=4 \pi \int_{\mathbb{R}_{+}} r \sin (k r) \overline{\phi_{\text {rad }}}(r) d r .
$$

which agrees with the FT for the spherically symmetric functions.) In practical calculations, one must first make the definite integral with respect to $r$, which gives a well-defined function of $k$. Then, integrating by parts with respect to $k$ one finally obtains

$$
\psi[\bar{\phi}]=\int_{\mathbb{R}_{+}} \frac{d k k^{2}}{(2 \pi)^{3}} \overline{\mathscr{F}_{\text {rad }}\{\phi\}}(k) \mathscr{F}_{D}\{\psi\}(k),
$$

thus indicating that the distributional FT $\mathscr{F}_{D}\{\psi\}(k)$ is also rotationally symmetric.

Although the tempered distribution $\psi[\phi]$ is defined in (13) as an expression independent of $M$, its final form explicitly includes the parameter $M$. It is therefore desirable to see how this presence effectively cancels itself, and this can be done by computing the derivative of the last line in (13) with respect to the parameter $M$

$$
\begin{aligned}
\frac{\partial}{\partial M^{2}} \psi[\phi] & =n \int_{\mathbb{R}^{3}}\left(M^{2}-\nabla_{k}^{2}\right)^{n-1} \overline{\mathscr{F}\{\phi\}}(\boldsymbol{k}) \mathscr{F}\left\{\frac{\psi}{\left(r^{2}+M^{2}\right)^{n}}\right\}(\boldsymbol{k}) \frac{d^{3} \boldsymbol{k}}{(2 \pi)^{3}} \\
& -n \int_{\mathbb{R}^{3}}\left(M^{2}-\nabla_{\boldsymbol{k}}^{2}\right)^{n} \overline{\mathscr{F}\{\phi\}}(\boldsymbol{k}) \mathscr{F}\left\{\frac{\psi}{\left(r^{2}+M^{2}\right)^{n+1}}\right\}(\boldsymbol{k}) \frac{d^{3} \boldsymbol{k}}{(2 \pi)^{3}},
\end{aligned}
$$

where in (16) one may push the derivative under the integral sign in $\mathscr{F}\left\{\psi /\left(r^{2}+M^{2}\right)^{n}\right\}$ that is given by a convergent integral. These two tempered distributions $\mathscr{S}^{\prime}\left(\mathbb{R}^{3}\right)$ are equal to each other, which can be easily checked by integrating by parts in the second case, where $\nabla_{k}^{2}$ can be inserted under the integral sign $\mathscr{F}\left\{\psi /\left(r^{2}+M^{2}\right)^{n+1}\right\}$, which is given by the convergent integral. This brings us to the expected result $\frac{\partial}{\partial M^{2}} \psi[\phi]=0$, but we discovered the crucial role played by integration by parts for checking the $M$-independence of $\psi[\bar{\phi}]$.

If one imposes the dilation transformation on the function $\psi(r) \rightarrow \psi^{\lambda}(r)=\psi(\lambda r)$, then the radial Parseval equation (17) for the distribution $\psi^{\lambda}[\phi]$ becomes

$$
\begin{aligned}
\psi^{\lambda}[\bar{\phi}] & =\int_{\mathbb{R}_{+}}\left(M^{2}-\partial_{k}^{2}\right)^{n}\left[k \overline{\mathscr{F}_{\text {rad }}\{\phi\}}(k)\right]\left[\int_{\mathbb{R}_{+}} \frac{r \sin (k r) \psi(r \lambda)}{\left(r^{2}+M^{2}\right)^{n}} d r\right] \frac{d k}{2 \pi^{2}} \\
& =\int_{\mathbb{R}_{+}}\left(M_{1}^{2}-\partial_{p}^{2}\right)^{n}\left[p \overline{\mathscr{F}_{\text {rad }}\{\phi\}}(p \lambda)\right]\left[\int_{\mathbb{R}_{+}} \frac{\xi \sin (p \xi) \psi(\xi)}{\left(\tilde{\mathcal{\zeta}}^{2}+M_{1}^{2}\right)^{n}} d \xi\right] \frac{d p}{2 \pi^{2}} \\
& =\int_{\mathbb{R}_{+}} \overline{\mathscr{F}_{\text {rad }}\{\phi\}}(p \lambda) \mathscr{F}_{D}\{\psi\}(p) \frac{d p p^{2}}{(2 \pi)^{3}}=\frac{1}{\lambda^{3}} \int_{\mathbb{R}_{+}} \overline{\mathscr{F}_{\text {rad }}\{\phi\}}(k) \mathscr{F}_{D}\{\psi\}\left(k \lambda^{-1}\right) \frac{d k k^{2}}{(2 \pi)^{3}}
\end{aligned}
$$

where we changed the integral variables: $r=\xi \lambda^{-1}, k=p \lambda$ and the parameter $M=M_{1} \lambda$. Thus, in the sense of distributions, we have the distributional FT for the dilation transformed function $\mathscr{F}_{D}\left\{\psi^{\lambda}\right\}(k)=\lambda^{-3} \mathscr{F}_{D}\{\psi\}\left(k \lambda^{-1}\right)$. However, if the function $\psi$ has dilation symmetry, then its distributional FT also has dilation symmetry

$$
\psi^{\lambda}(\boldsymbol{r})=\psi(\lambda \boldsymbol{r})=\lambda^{d} \psi(\boldsymbol{r}) \Longrightarrow \mathscr{F}_{D}\left\{\psi^{\lambda}\right\}(p)=\lambda^{-(d+3)} \mathscr{F}_{D}\{\psi\}(p) .
$$

This shows that our novel method of computing FT for functions in $\mathbb{R}^{3}$ does not conflict with rotational and dilational symmetry. 
Example 3. One may check the above tools for a toy function $W(x)=\frac{1}{r^{2}}$, which is both rotationally symmetric and dilation covariant $W^{\lambda}(\boldsymbol{x})=W(\lambda \boldsymbol{x})=\lambda^{-2} W(\boldsymbol{x})$ and $W \in L^{1}\left(\mathbb{R}^{3}\right)$, so its FT can be also computed from the standard definition (1)

$$
\mathscr{F}\{W\}(\boldsymbol{k})=\int_{\mathbb{R}^{3}} e^{-i \boldsymbol{k} \cdot \boldsymbol{x}} \frac{1}{r^{2}} d^{3} \boldsymbol{x}=\frac{4 \pi}{k} \int_{\mathbb{R}_{+}} \frac{\sin (k r)}{r} d r=\frac{2 \pi^{2}}{k} .
$$

Then, we used the Parseval equation (17) with $n=1$, for the functional $W[\phi]$, which gives the distribution, where first we performed the integral with respect to $r$, and then we integrated by parts with respect to $k$

$$
\begin{aligned}
W[\bar{\phi}] & =\int_{\mathbb{R}^{3}} \bar{\phi}(\boldsymbol{x}) W(\boldsymbol{x}) d^{3} \boldsymbol{x}=\int_{\mathbb{R}_{+}}\left(M^{2}-\partial_{k}^{2}\right)\left[k \overline{\mathscr{F}_{\text {rad }}\{\phi\}}(k)\right]\left[\int_{\mathbb{R}_{+}} \frac{\sin (k r)}{r\left(r^{2}+M^{2}\right)} d r\right] \frac{d k}{2 \pi^{2}} \\
& =\frac{1}{4 \pi} \int_{\mathbb{R}_{+}}\left(M^{2}-\partial_{k}^{2}\right)\left[k \overline{\mathscr{F}_{\text {rad }}\{\phi\}}(k)\right] \frac{1-e^{-M k}}{M^{2}} d k=\frac{1}{4 \pi} \int_{\mathbb{R}_{+}} k \mathscr{F}_{\text {rad }}\{\phi\}(k) d k,
\end{aligned}
$$

which implies $\mathscr{F}_{D}\{W\}(\boldsymbol{k})=\frac{2 \pi^{2}}{k}=\mathscr{F}\{W\}(\boldsymbol{k})$, so it agrees with the standard FT, as expected. Moreover, the dilation symmetry relation for FT is $\mathscr{F}\left\{W^{\lambda}\right\}(\boldsymbol{k})=\mathscr{F}\{W\}(\lambda \boldsymbol{k})=\lambda^{-1} \mathscr{F}\{W\}(\boldsymbol{k})$ in agreement with the Formula (23).

\section{Results for Coulomb-like Potentials}

Next, we calculated FT for Coulomb-type potentials in turn: the smooth potential $V_{P}(\boldsymbol{x})=\frac{1}{r} e^{-b^{2} / r}$, the soft-core potential $U_{a}(\boldsymbol{x})=\frac{1}{\sqrt{r^{2}+a^{2}}}$ for $a \in \mathbb{R}_{+}$, and the plain Coulomb potential $V_{c}(x)=\frac{1}{r}$. All of them are locally integrable in $\mathbb{R}^{3}$; thus, one can define the respective tempered distributions, for test function $\phi \in \mathscr{S}\left(\mathbb{R}^{3}\right)$,

$$
V_{P}[\bar{\phi}]=\int_{\mathbb{R}^{3}} \bar{\phi}(\boldsymbol{x}) V_{c}(\boldsymbol{x}) d^{3} \boldsymbol{x}, \quad U_{a}[\bar{\phi}]=\int_{\mathbb{R}^{3}} \bar{\phi}(\boldsymbol{x}) U_{a}(\boldsymbol{x}) d^{3} \boldsymbol{x}, \quad V_{c}[\bar{\phi}]=\int_{\mathbb{R}^{3}} \bar{\phi}(\boldsymbol{x}) V_{c}(\boldsymbol{x}) d^{3} \boldsymbol{x} .
$$

For $V_{P}[\bar{\phi}]$, we may take the simplest form of the Parseval equation (17) with $n=1$ and $M=0$, because the exponential factor smooths the vicinity of $r=0$; then, we computed the integral with respect to $r$ using formula (3.957.1) in [16]

$$
\begin{aligned}
& V_{P}[\bar{\phi}]=\int_{\mathbb{R}_{+}}\left(-\partial_{k}^{2}\right)[k \overline{\mathscr{F}} \text { rad }\{\phi\}(k)]\left[\int_{\mathbb{R}_{+}} \frac{d r}{2 \pi^{2}} \frac{\sin (k r)}{r^{2}} e^{-b^{2} / r} d r\right] \frac{d k}{2 \pi^{2}} \\
& =\frac{2}{\pi^{2} b} \int_{\mathbb{R}_{+}} \partial_{k}^{2}\left[k \overline{\mathscr{F}_{\text {rad }}\{\phi\}}(k)\right] \sqrt{k} \Im\left[e^{i \pi / 4} K_{1}\left(e^{i \pi / 4} b \sqrt{k}\right)\right] d k,
\end{aligned}
$$

where $K_{n}(z)=K_{-n}(z)$ is the modified Bessel function of the first kind of the n-th order. The integration by parts with respect to $k$ introduces no boundary terms and one needs to take the second order derivative for the modified Bessel function

$$
\frac{\partial^{2}}{\partial k^{2}} \sqrt{k} K_{1}(\alpha \sqrt{k})=\frac{\alpha^{2}}{4} \frac{1}{\sqrt{k}} K_{1}(\alpha \sqrt{k}) .
$$

Therefore, by analogy with (20), we obtain differential equation

$$
V_{P}[\bar{\phi}]=\int_{\mathbb{R}_{+}} \overline{\mathscr{F}_{\text {rad }}\{\phi\}}(k) F_{D}\left\{V_{P}\right\}(k) \frac{d k k^{2}}{(2 \pi)^{3}},
$$

with the distributional FT

$$
F_{D}\left\{V_{P}\right\}(k)=\frac{16 \pi}{k b} \frac{\partial^{2}}{\partial k^{2}} \sqrt{k} \Im\left[e^{i \pi / 4} K_{1}\left(e^{i \pi / 4} b \sqrt{k}\right)\right]=\frac{4 \pi b}{k \sqrt{k}} \Re\left[e^{i \pi / 4} K_{1}\left(e^{i \pi / 4} b \sqrt{k}\right)\right]
$$

This FT agrees with the corresponding result in [7], provided we use $\Im(i z)=\Re(z)$ and take into account the slightly different definition of FT therein. We also note that in [7] 
the damping factor $e^{-\kappa r}$ with $\kappa>0$ was added before computing the FT, and the limit of $\kappa \searrow 0$ was taken for the result obtained.

Then, we calculated the distributional FT for the soft-core Coulomb potential $U^{a}(\boldsymbol{x})=\frac{1}{\sqrt{r^{2}+a^{2}}}$, where we can take (17) with $n=1$ and $M=0$, which gives the distribution that allows to compute the integral with respect to $r$ by using formula (3.754.2) in [16]

$$
\begin{aligned}
U^{a}[\bar{\phi}] & =-\int_{\mathbb{R}_{+}} \partial_{k}^{2}\left[k \overline{\mathscr{F}_{\text {rad }}\{\phi\}}(k)\right]\left[\int_{\mathbb{R}_{+}} \frac{1}{\sqrt{r^{2}+a^{2}}} \frac{\sin (k r)}{r} d r\right] \frac{d k}{2 \pi^{2}} \\
& =-\int_{\mathbb{R}_{+}} \partial_{k}^{2}\left[k \overline{\mathscr{F}_{\text {rad }}\{\phi\}}(k)\right]\left[\int_{0}^{k} K_{0}(a p) d p\right] \frac{d k}{2 \pi^{2}} .
\end{aligned}
$$

The integration by parts with respect to $k$ introduces no boundary terms; therefore, we obtained

$$
U^{a}[\phi]=\int_{\mathbb{R}_{+}} \overline{\mathscr{F}_{\text {rad }}\{\phi\}}(k) F_{D}\left\{U^{a}\right\}(k) \frac{d k k^{2}}{(2 \pi)^{3}}
$$

with the distributional FT

$$
F_{D}\left\{U^{a}\right\}(k)=-\frac{4 \pi}{k} \frac{\partial^{2}}{\partial k^{2}} \int_{0}^{k} K_{0}(a p) d p=\frac{4 \pi}{k} a K_{1}(a k) .
$$

For this soft-core Coulomb potential we may make alternative calculations that begin with separation of the leading term (for $r \gg a$ ) as $U^{a}(\boldsymbol{x})=V_{c}(\boldsymbol{x})+U_{s u b}^{a}(\boldsymbol{x})$, where the subtracted soft-core potential $U_{\text {sub }}^{a}(x)$ follows from the relation

$$
U_{s u b}^{a}(x)=\frac{1}{\sqrt{r^{2}+a^{2}}}-\frac{1}{r}=-\int_{0}^{a} \frac{\mu}{\left(r^{2}+\mu^{2}\right)^{3 / 2}} d \mu .
$$

This leads to the subtracted functional

$$
\begin{aligned}
U_{\text {sub }}^{a}[\bar{\phi}] & =-\int_{\mathbb{R}_{+}}\left(M^{2}-\partial_{k}^{2}\right)\left[k \overline{\mathscr{F}_{\text {rad }}\{\phi\}}(k)\right]\left[\int_{0}^{a} \int_{\mathbb{R}_{+}} \frac{\mu r}{\left(r^{2}+\mu^{2}\right)^{3 / 2}} \frac{\sin (k r)}{r^{2}+M^{2}} d \mu d r\right] \frac{d k}{2 \pi^{2}} \\
& =-\int_{\mathbb{R}_{+}} k \overline{\mathscr{F}_{\text {rad }}\{\phi\}}(k)\left[\int_{0}^{a} \int_{\mathbb{R}_{+}} \frac{\mu r \sin (k r)}{\left(r^{2}+\mu^{2}\right)^{3 / 2}} d r d \mu\right] \frac{d k}{2 \pi^{2}}
\end{aligned}
$$

where we integrated by parts twice with respect to $k$, inserting the differentiation under the sign of the integral. Then, the integrals with respect to $r$ and $\mu$ can be calculated successively, using formula (3.754.3) in [16]

$$
\int_{0}^{a} \int_{\mathbb{R}_{+}} \frac{\mu r \sin (k r)}{\left(r^{2}+\mu^{2}\right)^{3 / 2}} d r d \mu=k \int_{0}^{a} \mu K_{0}(\mu k) d \mu=-a K_{1}(a k)+\frac{1}{k},
$$

giving the final form of the subtracted functional

$$
U_{\text {sub }}^{a}[\bar{\phi}]=\int_{\mathbb{R}_{+}} k \overline{\mathscr{F}_{\text {rad }}\{\phi\}}(k)\left(a K_{1}(a k)-\frac{1}{k}\right) \frac{d k}{2 \pi^{2}}
$$

and the distributional FT for $U_{s u b}^{a}$

$$
\mathscr{F}_{D}\left\{U_{\text {sub }}^{a}\right\}(k)=\frac{4 \pi}{k}\left(a K_{1}(a k)-\frac{1}{k}\right) .
$$

From the formula $U^{a}(x)=V_{c}(x)+U_{\text {sub }}^{a}(x)$, one directly obtains the formula for the functionals $V_{c}[\phi]=U^{a}[\phi]-U_{s u b}^{a}[\phi]$ and next the formula for FTs

$$
\mathscr{F}_{D}\left\{V_{c}\right\}(k)=\mathscr{F}_{D}\left\{U^{a}\right\}(k)-\mathscr{F}_{D}\left\{U_{s u b}^{a}\right\}(k)=\frac{4 \pi}{k^{2}} .
$$


In this way, we obtained the distributional FT for the Coulomb potential, which agrees with the expression obtained earlier by different methods. This clearly shows that a FT that is symmetric under dilation can appear as a difference of two FTs that are not symmetric under dilation.

At last, for checking consistency, we calculated directly the distributional FT for the Coulomb potential, so we took (17) with $n=1$ and $M>0$,

$$
V_{c}[\phi]=\int_{\mathbb{R}_{+}}\left(M^{2}-\partial_{k}^{2}\right)\left[k \overline{\mathscr{F}_{\text {rad }}\{\phi\}}(k)\right]\left[\int_{\mathbb{R}_{+}} \frac{\sin (k r)}{r^{2}+M^{2}} d r\right] \frac{d k}{2 \pi^{2}},
$$

and, as before, first we needed to calculate the integral with respect to $r$. Using formula (8.217.1) in [16], we obtained

$$
\int_{\mathbb{R}_{+}} \frac{\sin (k r)}{r^{2}+M^{2}} d r=\frac{1}{2 M} F_{-}(M k), \quad F_{ \pm}(x):=e^{-x} \operatorname{Ei}(x) \pm e^{x} \operatorname{Ei}(-x)
$$

where $\operatorname{Ei}(z)$ is the exponential integral function and then we may integrate by parts with respect to $k$

$$
\begin{aligned}
V_{c}[\bar{\phi}] & =\frac{1}{4 \pi^{2} M} \int_{\mathbb{R}_{+}}\left(M^{2}-\partial_{k}^{2}\right)\left[k \overline{\mathscr{F}_{\text {rad }}\{\phi\}}(k)\right] F_{-}(k M) d k \\
& =-\frac{1}{2 \pi^{2}} \int_{\mathbb{R}_{+}} \partial_{k}\left[k \overline{\mathscr{F}_{\text {rad }}\{\phi\}}(k)\right] \ln (M k) d k=\frac{1}{2 \pi^{2}} \int_{\mathbb{R}_{+}} \overline{\mathscr{F}_{\text {rad }}\{\phi\}}(k) d k .
\end{aligned}
$$

(Above we have used the differential properties of $F_{ \pm}$, that are discussed in Appendix B). Thus, the distributional FT is $\mathscr{F}_{D}\left\{V_{c}\right\}(k)=\frac{4 \pi}{k^{2}}$, which agrees with the previous result (35).

The distributional FT for the pure Coulomb potential $V_{c}$ can be used to prove the Poisson equation for $V_{c}$ in the sense of distribution $\mathscr{S}^{\prime}\left(\mathbb{R}^{3}\right)$. To demonstrate this, we calculates a new distribution $V_{c}\left[\nabla^{2} \bar{\phi}\right]$ for test function $\phi \in \mathscr{S}\left(\mathbb{R}^{3}\right)$, which allows for performing distributional derivatives, applying the Parseval equation for tempered distributions and the definition of Dirac delta singular function, successively

$$
\begin{aligned}
V_{c}\left[\nabla^{2} \bar{\phi}\right] & :=\int_{\mathbb{R}^{3}} \nabla^{2} \bar{\phi}(\boldsymbol{x}) V_{c}(\boldsymbol{x}) d^{3} \boldsymbol{x}=\int_{\mathbb{R}^{3}} \overline{\mathscr{F}\left\{\boldsymbol{\nabla}^{2} \phi\right\}}(\boldsymbol{k}) \mathscr{F}_{D}\left\{V_{c}\right\}(k) \frac{d^{3} \boldsymbol{k}}{(2 \pi)^{3}} \\
& =\int_{\mathbb{R}^{3}}\left(-\boldsymbol{k}^{2}\right) \overline{\mathscr{F}\{\phi\}}(\boldsymbol{k}) \frac{4 \pi}{k^{2}} \frac{d^{3} \boldsymbol{k}}{(2 \pi)^{3}}=-4 \pi \int_{\mathbb{R}^{3}} \overline{\mathscr{F}\{\phi\}}(\boldsymbol{k}) \frac{d^{3} \boldsymbol{k}}{(2 \pi)^{3}} \\
& =-4 \pi \bar{\phi}(\mathbf{0})=-4 \pi \int_{\mathbb{R}^{3}} \bar{\phi}(\boldsymbol{x}) \delta^{3}(\boldsymbol{x}) d^{3} \boldsymbol{x} .
\end{aligned}
$$

Thus, we may integrate by parts, which leads to distributional derivatives,

$$
\int_{\mathbb{R}^{3}} \nabla^{2} \bar{\phi}(x) V_{c}(x) d^{3} x=\int_{\mathbb{R}^{3}} \bar{\phi}(x) \bar{\nabla}_{D}^{2} V_{c}(x) d^{3} x=-4 \pi \int_{\mathbb{R}^{3}} \bar{\phi}(x) \delta^{3}(\boldsymbol{x}) d^{3} x
$$

so, in the sense of distributions $\mathscr{S}^{\prime}\left(\mathbb{R}^{3}\right)$, we draw as a conclusion the Poisson equation, with distributional derivatives, for the Coulomb potential [2]

$$
\bar{\nabla}_{D}^{2} V_{c}(x)=-4 \pi \delta^{3}(x) .
$$

\section{Discussion and Further Research}

The proposed novel method of computing FT for slowly increasing functions can be applied to functions that are locally integrable and can be tempered with a polynomial of a finite order. The most troublesome part of this procedure is computation of a convergent improper integral, which may additionally depend on some arbitrary auxiliary parameterin this study, we used the formulas for integrals from the table of integrals [16]. 
Due to the rotational symmetry of the analyzed functions, we obtained radial integrals and radial test functions. The dilation symmetry manifested itself in a less evident way but was still not broken at any stage of the calculation.

For the softened Coulomb potential $V_{p}(x)$ and the soft-core Coulomb potential $U^{a}(x)$, due to a smooth dependence on $r \rightarrow 0$, the Parseval equation may be taken with $M=0$, which simplifies our computation and leads to differentiable auxiliary functions. Then, the distributional FT for each potential becomes the second-order derivative of the respective auxiliary function. Both the auxiliary functions and the FTs depend on the modified Bessel functions. However, the plain Coulomb potential $V_{c}(\boldsymbol{x})$ is singular for $r \rightarrow 0$, so we needed to take $M \neq 0$, and further steps of computation are more difficult. We obtained the auxiliary function that depends on the exponential integral function of $\operatorname{Ei}(z)$. However, it appeared only in an intermediate step of the computation, while in the final result this special function disappeared.

Furthermore, we showed the usefulness of calculating the FT for a subtracted function, $U_{\text {sub }}^{a}(x)$, that is already an integrable function, at the cost of introducing an additional proper integral with respect to an auxiliary parameter, in our case $\mu$.

In general, it can be said that the increased rigor of the method comes at a price, as the calculations become somewhat more complicated. The applicability of our new method, according to the lemma in Section 2, is limited to locally integrable functions on $\mathbb{R}^{3}$ that grow slowly as $|x| \rightarrow \infty$. However, these functions can also contain additional variables that are not integrated when computing FT. In physics, such an additional variable might be a time coordinate. For example, in quantum field theory, our method can be used for calculation of the Wightman functions [17], for a free scalar field. The resulting expression has the Lorentz symmetry and contains distributional derivatives - this will be published soon in a separate article. This new result deviates from previous approaches, where one either uses mathematically inaccurate tricks, as in [18], or introduces damping factors that break an important physical symmetry (here, the Lorentz symmetry) [19] or uses Lorentz symmetry to analyze divergent integrals [20]. The main feature of the new expressions for Wightman functions is the appearance of distributional derivatives, which has not been used before. We plan to apply our novel approach to FT calculations in the context of light front quantization [21], where existing previous results for Wightman functions [22,23] should be checked and/or improved by analysis from a different point of view. Our computational method can be applied to other mathematical problems arising in quantum mechanics that require the computation of FT for functions that are not in $L^{1}\left(\mathbb{R}^{n}\right)$; for example, regarding the time-evolution operator in the free Schrödinger equation, see [24].

We also need to comment on the FT calculated for the spherically symmetric generalized function $r^{\lambda}$ for $\lambda \neq-n,-n-2, \ldots$ in $\mathbb{R}^{n}$ in [12]. A key part of this calculation is the dilation covariance (homogeneity), so it cannot be used for the case of Coulomb potentials considered in this study. Therefore, we plan to apply our novel procedure to compute the FT for the above generalized function in a separate study, which should provide a basis for comparing the two methods.

Author Contributions: Conceptualization, J.A.P.; methodology, J.A.P.; validation, E.D.-C. and J.A.P.; formal analysis, E.D.-C. and J.A.P.; investigation, E.D.-C. and J.A.P.; writing-original draft preparation, E.D.-C. and J.A.P.; writing-review and editing, J.L.C. and J.A.P.; funding acquisition, J.L.C. All authors have read and agreed to the published version of the manuscript.

Funding: This research received no external funding.

Institutional Review Board Statement: Not applicable.

Informed Consent Statement: Not applicable.

Data Availability Statement: Not applicable.

Conflicts of Interest: The authors declare no conflict of interest. 


\section{Appendix A. Equivalence of Two Calculation Methods}

For proving the equivalence of two calculation methods, we may begin with (16) for $n=1$ and $M>0$. First we may exponentiate the dominator by means of the additional integral

$$
\frac{1}{r^{2}+M^{2}}=\frac{1}{M} \int_{\mathbb{R}_{+}} \sin (M \alpha) e^{-\alpha r} d \alpha,
$$

thus we obtain

$$
\begin{aligned}
\mathscr{F}\left\{\psi /\left(r^{2}+M^{2}\right)\right\}(\boldsymbol{k}) & =\frac{4 \pi}{k} \int_{\mathbb{R}_{+}} r \sin (k r) \psi(r)\left[\int_{\mathbb{R}_{+}} e^{-\alpha r} \frac{\sin (M \alpha)}{M} d \alpha\right] d r \\
& =\frac{4 \pi}{k} \int_{\mathbb{R}_{+}} \frac{\sin (M \alpha)}{M}\left[\int_{\mathbb{R}_{+}} r \sin (k r) \psi(r) e^{-\alpha r} d r\right] d \alpha,
\end{aligned}
$$

where Fubini's theorem permits to switch the order of integrations. Then, the action of $\left(M^{2}-\nabla^{2}\right)$ gives

$$
\begin{aligned}
& \left(M^{2}-\nabla^{2}\right) \mathscr{F}\left\{\psi /\left(r^{2}+M^{2}\right)\right\}(\boldsymbol{k})= \\
& =4 \pi \int_{\mathbb{R}_{+}} \frac{\sin (M \alpha)}{M}\left(M^{2}+\frac{\partial^{2}}{\partial \alpha^{2}}\right)\left[\int_{\mathbb{R}_{+}} r \frac{\sin (k r)}{k} \psi(r) e^{-\alpha r} d r\right] d \alpha \\
& \quad=\lim _{\alpha \searrow 0} \int_{\mathbb{R}_{+}} r \frac{\sin (k r)}{k} \psi(r) e^{-\alpha r} d r,
\end{aligned}
$$

where we used $\nabla_{k}^{2} \sin (k r) / k=-r^{2} \sin (k r) / k$ and integrated by parts with respect to $\alpha$.

This calculation clearly shows that the two computational methods give the same results if the distributional partial derivatives that appear in (14) are essentially standard partial derivatives; otherwise, only the second method, the one proposed in this study, gives the correct answer.

\section{Appendix B. Differential Properties of the Exponential Integral Function}

Series representation given by (8.214.) in [16], for $x \in \mathbb{R}$

$$
\operatorname{Ei}(x)=\gamma_{E}+\ln |x|+\sum_{n=1}^{\infty} \frac{x^{n}}{n \cdot n !}, \quad \widetilde{\operatorname{Ei}}(x):=\sum_{n=1}^{\infty} \frac{x^{n}}{n \cdot n !}
$$

leads to derivatives

$$
\frac{d}{d x} \widetilde{\operatorname{Ei}}(x)=\frac{e^{x}-1}{x}, \quad \frac{d}{d x} \widetilde{\operatorname{Ei}}(-x)=\frac{e^{-x}-1}{x} .
$$

Thus, for $\widetilde{F}_{ \pm}(x):=e^{-x} \widetilde{\operatorname{Ei}}(x) \pm e^{x} \widetilde{\operatorname{Ei}}(-x)$, we found the derivatives

$$
\frac{d}{d x} \widetilde{F}_{-}(x)=-\widetilde{F}_{+}(x)+2 \frac{\sinh (x)}{x}, \quad \frac{d}{d x} \widetilde{F}_{+}(x)=-\widetilde{F}_{-}(x)+2 \frac{1-\cosh (x)}{x},
$$

Thus, the derivative of $F_{-}(x)=\widetilde{F}_{-}(x)-2 \sinh (x)\left(\gamma_{E}+\ln |x|\right)$ is

$$
\frac{d}{d x} F_{-}(x)=-\widetilde{F}_{+}(x)-2 \cosh (x)\left(\gamma_{E}+\ln |x|\right)=-F_{+}(x)
$$

We calculated the derivative of the regularized function $F_{+}^{r e g}(x):=F_{+}(x)-2 \ln |x|$

$$
\frac{d}{d x} F_{+}^{r e g}(x)=-\widetilde{F}_{-}(x)+2 \sinh (x)\left(\gamma_{E}+\ln |x|\right)=-F_{-}(x)
$$


So, we found derivatives

$$
\frac{d}{d x} F_{-}(x)=-F_{+}^{r e g}(x)-2 \ln |x|, \quad \frac{d}{d x} F_{+}^{r e g}(x)=-F_{-}(x),
$$

which can be used for doing partial integration in the linear functionals

$$
\frac{M}{2} \int_{\mathbb{R}_{+}}[k g(k)]^{\prime \prime} F_{-}(k M) d k=\int_{\mathbb{R}_{+}} k g(k) F_{-}(k M) d k+\int_{\mathbb{R}_{+}}[k g(k)]^{\prime} \ln (M k) d k,
$$

where primes denote derivatives with respect to $k$.

\section{References}

1. Zemanian, A.H. Distribution Theory and Transform Analysis, 1st ed.; McGraw-Hill Book Company, Inc.: New York, NY, USA, 1965; pp. 171-191.

2. Jackson, J.D. Classical Electrodynamics, 1st ed.; John Wiley and Sons, Inc.: New York, NY, USA; London, UK; Sydney, Australia, 1962; pp. 12-14.

3. Reiher, M.; Wolf, A. Relativistic Quantum Chemistry, 2nd ed.; Wiley-VCH Verlag and Co. KGaA: Weinheim, Germany, 2015; pp. 653-656.

4. Adkins, G.S. Three-dimensional Fourier transforms, integrals of spherical Bessel functions, and novel delta function identities. arXiv 2013, arXiv:1302.1830.

5. Straton, J.C. General state-to-state transitions in atoms for either Yukawa or Coulomb potentials via Fourier transforms. Can. J. Phys. 2015, 93, 326-338. [CrossRef]

6. Bobrov, V.B.; Trigger, S.A.; Zagorodny, A.G. On the Fourier Transform Features for the Coulomb Potential in Statistical Theory. Bull. Lebedev Phys. Inst. 2015, 42, 329-332. [CrossRef]

7. Stachura, E. Existence of propagators for time-dependent Coulomb-like potentials. Rocky Mt. J. Math. 2019, 49, 2347-2374. [CrossRef]

8. Iñarrea, M.; Lanchares, V.; Palacián, J.F.; Pascual, A.I.; Salas, J.P.; Yanguas, P. Effects of a soft-core Coulomb potential on the dynamics of a hydrogen atom near a metal surface. Commun. Nonlinear Sci. Numer. Simul. 2019, 68, 94-105. [CrossRef]

9. Hall, R.L.; Saad, N.; Sen, K.D.; Ciftci, H. Energies and wave functions for a soft-core Coulomb potential. Phys. Rev. A 2009, 80, 032507. [CrossRef]

10. Hall, R.L.; Zorin, P. Dirac eigenvalues for a softcore Coulomb potential in d dimensions. J. Phys. A Math. Theor. 2012, 45, 115303. [CrossRef]

11. Agboola, D.; Zhang, Y.-Z. Quasi-exactly solvable relativistic soft-core Coulomb models. Ann. Phys. 2012, 327, 2275-2287. [CrossRef]

12. Gel'fand, I.M.; Shilov, G.E. Generalized Functions. Volume 1. Properties and Operations; Translated from the 1958 Russian original by Eugene Saletan; Academic Press: New York, NY, USA; London, UK, 1964; pp. 192-195. [CrossRef]

13. Zeidler, E. Quantum Field Theory I. Basics in Mathematics and Physics, 1st ed.; Springer: Berlin/Heidelberg, Germany, 2009; pp. 537-539.

14. Kanwal, R.P. Generalized Functions: Theory and Techniques; Birkhäuser: Boston, MA, USA, 1998; pp. 138-148

15. Blanchard, P.; Brüning, E. Mathematical Methods in Physics, 2nd ed.; Birkhäuser: Boston, MA, USA; Springer: Cham, Switzerland, 2015; pp. 133-162

16. Gradshteyn, I.S.; Ryzhik, I.M. Table of Integrals, Series, and Products, 6th ed.; Academic Press: San Diego, CA, USA, 2000.

17. Wightman, A.S. Quantum Field Theory in Terms of Vacuum Expectation Values. Phys. Rev. 1956, 101, 860-866.

18. Schweber, S. An Introduction to Relativistic Quantum Field Theory; Dover Publications, Inc.: Mineola, NY, USA, 2005; pp. 181-183. [CrossRef]

19. Bogoliubov, N.N.; Logunov, A.A.; Oksak, A.I.; Todorov, I.T. General principles of Quantum Field Theory; Kluwer Academic Publishers: Dordrecht, The Netherlands, 1990; p. 354.

20. Liu, D.; Chen, F.; Chen, S.; Ma, N. Calculating Pauli-Jordan function. Eur. J. Phys. 2020, 41, 035406.

21. Burkardt, M. Light front quantization. Adv. Nucl. Phys 1996, 23, 1-74. [CrossRef]

22. Przeszowski, J.A. Lorentz symmetry for the light-front Wightman functions. Acta Phys. Pol. Proc. Suppl. 2013, 6, 327-333.

23. Przeszowski, J.A.; Żochowski, J. Scale and Lorentz transformations at the light-front. Few Body Syst. 2014, 55, 485-491. [CrossRef]

24. Teschl, G. Mathematical Methods in Quantum Mechanics, with Applications to Schrödinger Operators; Graduate Studies in Math. 99; American Mathematical Society: Providence, RI, USA, 2009; pp. 139-147. [CrossRef] 